\title{
Estrategias para el control de la frecuencia cardíaca en pacientes con fibrilación auricular
}

\section{Strategies for control of heart rate in patients with atrial fibrillation}

\author{
Alberto Negrete-Salcedo ${ }^{\mathrm{a}, \mathrm{b}, \mathrm{c}, *}$ y Efraín Gil-Roncancio ${ }^{\mathrm{a}, \mathrm{b}, \mathrm{c}}$ \\ a Servicio de Electrofisiología, Centro Médico Imbanaco, Cali, Colombia \\ b Clínica de Occidente, Cali, Colombia \\ c Clínica Castellana, Cali, Colombia
}

Recibido el 3 de octubre de 2016; aceptado el 6 de octubre de 2016

Disponible en Internet el 8 de noviembre de 2016

Cuando no es posible mantener el ritmo sinusal, uno de los objetivos es controlar la respuesta ventricular dentro de un rango razonable mientras que la fibrilación persiste. Esto se conoce como «control de la frecuencia». Tal estrategia bien implementada tiene efectos benéficos para el paciente pues mejora la calidad de vida y reduce la morbilidad y la posibilidad de desarrollar taquicardiomiopatía ${ }^{1}$.

La mayoría de pacientes con fibrilación auricular requiere medicamentos para disminuir la respuesta ventricular. Es necesario hacerlo ya que las frecuencias altas empeoran los síntomas. Cuando una persona entra en ritmo de fibrilación auricular y no recibía previamente ningún medicamento antiarrítmico, generalmente tiene una frecuencia que oscila entre 110 y 125 latidos por minuto ${ }^{2}$. Adicionalmente, estudios recientes relacionan la falta del control de la frecuencia cardíaca con la progresión de la enfermedad hacia formas sostenidas de la misma ${ }^{3}$.

La fibrilación auricular puede producir síntomas y alteraciones funcionales cardiovasculares por varios mecanismos. En primer lugar está la pérdida de la contribución auricular en el llenado ventricular, la cual es más notoria cuando la frecuencia cardíaca supera los 100 latidos por

\footnotetext{
* Autor para correspondencia.

Correo electrónico: alberto.negrete@imbanaco.com.co (A. Negrete-Salcedo).
}

minuto ${ }^{4}$, debido a que mientras la frecuencia aumenta, el tiempo de llenado diastólico disminuye. Dado que muchos de los pacientes que desarrollan esta arritmia tienen otras patologías coexistentes que causan disfunción diastólica como hipertensión arterial, enfermedad coronaria, diabetes mellitus o enfermedad valvular, el llenado ventricular puede tener un efecto crítico en el mantenimiento de una fracción de eyección adecuada. Es por eso que en algunos casos los aumentos leves de la frecuencia pueden relacionarse con síntomas graves de falla cardíaca, incluso con edema pulmonar o colapso hemodinámico.

El desarrollo de disfunción ventricular en pacientes sometidos crónicamente a frecuencias altas, recibe el nombre de «miocardiopatía inducida por taquicardia ${ }^{5}$ o «taquicardiomiopatía», que es una alteración que produce disminución progresiva de la fracción de eyección del ventrículo izquierdo, puede asociarse con aumento en el tamaño de las cavidades y llevar a diferentes grados de insuficiencia mitral funcional, en un círculo vicioso con deterioro progresivo de las condiciones del enfermo. A pesar de lo grave de los cambios, se ha demostrado que el control adecuado de la frecuencia, puede revertir el daño miocárdico y en algunos casos se han visto mejorías dramáticas en la función ventricular después de un tratamiento apropiado ${ }^{6}$.

El control de la frecuencia es la conducta más usada por los médicos que tratan pacientes con fibrilación auricular, hecho que se debe a consideraciones de índole práctico, 
ya que es la estrategia más simple y menos costosa. En países o regiones con limitaciones económicas y geográficas el paciente suele consultar en etapas muy avanzadas de su enfermedad, siendo el control de la frecuencia la única alternativa práctica. Además de la patología de base que pueda tener el paciente, la fibrilación auricular produce cambios eléctricos y funcionales en sus aurículas que hacen que ésta se perpetúe, habiéndose comprobado que «la fibrilación auricular genera más fibrilación auricular». A pesar de que algunos pacientes pueden salir nuevamente a ritmo sinusal, es común que la fibrilación auricular se reitere con el paso del tiempo y por eso tengan síntomas recurrentes.

Durante el tratamiento es importante establecer si el control de la frecuencia ha sido adecuado. Algunos autores proponen mantener la frecuencia un poco por encima de la que tienen grupos de control en ritmo sinusal, aduciendo alteraciones del llenado ventricular. Además de mantener control sobre la frecuencia en reposo, un tratamiento adecuado debe brindar control sobre la frecuencia en ejercicio. Al respecto se sabe que los betabloqueadores como el verapamilo y el diltiazem son más efectivos que la digoxina durante el ejercicio. Así mismo, recientemente se ha encontrado evidencia suficiente que plantea un riesgo aumentado de mortalidad en pacientes con fibrilación auricular en quienes se utiliza digoxina ${ }^{7,8}$, de modo que el uso del medicamento debe limitarse para casos específicos con vigilancia estricta de la digoxemia, las interacciones farmacológicas y las alteraciones electrolíticas. Se recomienda mantener el paciente con fibrilación auricular dentro de las siguientes estrategias $^{9}$.

\section{Estrategia farmacológica (tabla 1)}

Obtener frecuencias cardiacas en reposo en cifras inferiores a 80 latidos por minuto e inferiores a 100 latidos por minuto durante ejercicio son los parámetros que se escogen en la mayoría de estudios clínicos incluyendo el AFFIRM y se alcanzan con el tratamiento médico en cerca del $60 \%$ de los $\operatorname{casos}^{10}$.

- En reposo: frecuencia entre 60-80 latidos por minuto.

- En ejercicio: frecuencia entre 90-115 latidos por minuto.

- En Holter: promedio inferior a 100 latidos por minuto.

- Durante procesos sobreagregados y estados descompensados: menos de 110 lpm.

Infortunadamente, en el estudio AFFIRM se encontró que un porcentaje importante de pacientes (22-37\%) no lograba permanecer dentro de los rangos esperados de frecuencia cardíaca, pese a todos los esfuerzos terapéuticos.

\section{Betabloqueadores}

Son los medicamentos más utilizados para este fin; en el estudio AFFIRM fueron los más efectivos ya que controlaron la respuesta ventricular en un $70 \%$ vs. $54 \%$ para calcioantagonistas. Además se pueden asociar con digoxina en pacientes con falla cardiaca.

\section{Calcioantagonistas no dihidropiridínicos}

Diltiazem y verapamilo son los más empleados y se dispone de ellos por vía oral. Disminuyen la respuesta ventricular en reposo y tras ejercicio, pero tienen limitaciones pues no se recomienda ampliamente su asociación con betabloqueadores o su uso en pacientes con falla cardiaca descompensada ni en aquellos con síndrome de pre-excitación ya pueden acelerar la conducción anterógrada por el haz anómalo y precipitar episodios de fibrilación ventricular.

\section{Digoxina}

Hoy en día su uso ha decrecido y es una terapia de segunda línea; su inicio de acción es lento, así que no se utiliza para el control rápido de la respuesta ventricular debido a que ejerce su efecto por aumento del tono parasimpático de base y limita su efecto durante el ejercicio, estrés fisiológico u otros periodos de incremento del tono simpático. Administrado por vía oral disminuye la respuesta ventricular en reposo pero no durante ejercicio; se puede combinar con betabloqueadores y calcioantagonistas y también utilizarse en pacientes con falla cardiaca descompensada. Su ventana terapéutica se estrecha en pacientes con falla renal, en ancianos y con el uso concomitante de calcioantagonistas y amiodarona, que aumentan los niveles séricos, en cuyos casos se recomienda titular los niveles en sangre ya que cifras mayores a $0,9 \mathrm{ng} / \mathrm{ml}$ se asocian con incremento de la mortalidad.

\section{Amiodarona}

Sus potenciales efectos tóxicos, las interacciones farmacológicas y el hecho de no ser superiores en el control de la respuesta ventricular a los betabloqueadores o a los calcioantagonistas, limitan su uso en esta indicación. Antes de usar este medicamento se deben considerar los riesgos: se sabe que presenta efecto proarrítmico incluyendo taquicardia ventricular polimorfa, y por sus efectos secundarios se debe descontinuar hasta en un $30 \%$ de los casos. Los más frecuentes son toxicidad tiroidea, fotosensibilidad y alteración de los niveles de anticoagulación debido a su interacción con la warfarina.

Una frecuencia ventricular promedio menor de 60 latidos por minuto sugiere disfunción del nodo aurículo-ventricular o un efecto inadecuado de medicamentos como betabloqueadores, digoxina, calcioantagonistas, clonidina y amiodarona entre otros. Si el ritmo de base es de fibrilación auricular y los intervalos R-R se observan regulares, el diagnóstico es bloqueo aurículo-ventricular completo.

\section{Estrategia no farmacológica: ablación del nodo aurículo-ventricular}

Es una estrategia altamente efectiva pero debe reservarse para pacientes refractarios al tratamiento farmacológico, pues implica implante de marcapasos con dependencia del mismo. Se reserva su uso en pacientes ancianos o que han desarrollado taquicardiomiopatía y en quienes la posibilidad de éxito de ablación curativa de la fibrilación auricular es 
Tabla 1 Medicamentos para uso parenteral en el control de la respuesta ventricular de pacientes con fibrilación auricular (útiles también en flutter y taquicardia atrial)

\begin{tabular}{|c|c|c|}
\hline Medicamento & Dosis & Presentación \\
\hline $\begin{array}{l}\text { Amiodarona para uso } \\
\text { parenteral }\end{array}$ & $\begin{array}{l}\text { Esquema convencional: } \\
150 \mathrm{mg} \text { en } 100 \mathrm{ml} \text { D5\% para } 10 \text { minutos. } \\
\text { Luego preparar una mezcla de } 900 \mathrm{mg} \text { en } 500 \mathrm{ml} \text { D5\%, de los } \\
\text { cuales se pasan en las siguientes } 6 \text { horas } 200 \mathrm{ml} \text { y en las } 18 \\
\text { restantes } 300 \mathrm{ml} \text { (dosis total de ataque }=1050 \mathrm{mg} \text { en } 24 \text { horas) } \\
\text { Esquema simplificado: } \\
\text { Bolo: } 5 \mathrm{mg} / \mathrm{kg} \text { en } 250 \mathrm{ml} \text { de D5\% en } 20 \text { - } 120 \text { minutos } \\
\text { Mantenimiento: } 10 \mathrm{mg} / \mathrm{kg} / \text { día para infusión continua en D5\% }\end{array}$ & $\begin{array}{l}\text { Ampollas de } 3 \mathrm{ml}=150 \mathrm{mg} \text { (50 } \\
\mathrm{mg} / \mathrm{ml})\end{array}$ \\
\hline Digoxina & $\begin{array}{l}\text { Aplicación rápida de } 0,5-1 \mathrm{mg} \text { IV y luego } 0,25 \mathrm{mg} \text { cada } 2-4 \\
\text { horas, para un total en } 24 \text { horas menor de } 1,5 \mathrm{mg}\end{array}$ & Ampollas de $1 \mathrm{ml}=0,25 \mathrm{mg}$ \\
\hline Esmolol & $\begin{array}{l}\text { Bolo: } 500 \text { microgramos } / \mathrm{kg} / \mathrm{min} \text { durante } 1 \text { minuto y luego } \\
50 \text { microgramos } / \mathrm{kg} / \mathrm{min} \text { durante } 4 \text { minutos } \\
\text { Mantenimiento: titular entre } 50-200 \text { microgramos } / \mathrm{kg} / \mathrm{min}\end{array}$ & $\begin{array}{l}\text { Ampollas de } 10 \mathrm{ml}=2500 \mathrm{mg} \\
(250 \mathrm{mg} / \mathrm{ml}) \text { (solo para usar en } \\
\text { infusión continua y diluido) }\end{array}$ \\
\hline Metoprolol & $\begin{array}{l}\text { Bolo: } 2,5-5 \mathrm{mg} \text { IV cada } 10 \text { minutos, máximo } 15 \mathrm{mg} \\
\text { Mantenimiento: } 5-10 \mathrm{mg} \text { IV cada } 12 \text { horas o } 25-100 \mathrm{mg} \text { VO cada } \\
12 \text { horas }\end{array}$ & $\begin{array}{l}\text { Ampollas de } 5 \mathrm{ml}=5 \mathrm{mg} \\
(1 \mathrm{mg} / \mathrm{ml})\end{array}$ \\
\hline Verapamilo & $\begin{array}{l}\text { Bolo: } 2,5-10 \mathrm{mg} \text { IV en } 2-5 \text { minutos } \\
\text { Mantenimiento: } 2,5-10 \mathrm{mg} \text { IV cada } 4-6 \text { horas o } 40-120 \mathrm{mg} \text { Vo } \\
\text { cada } 8 \text { horas }\end{array}$ & $\begin{array}{l}\text { Ampollas de } 2 \mathrm{ml}=5 \mathrm{mg} \\
(2,5 \mathrm{mg} / \mathrm{ml})\end{array}$ \\
\hline
\end{tabular}

Tabla 2 Recomendaciones

\begin{tabular}{|c|c|c|}
\hline Recomendación & Clase & Evidencia \\
\hline $\begin{array}{l}\text { Suministrar betabloqueadores } \\
\text { o calcio-antagonistas para } \\
\text { controlar la respuesta } \\
\text { ventricular en cualquier tipo } \\
\text { de fibrilación auricular }\end{array}$ & I & B \\
\hline $\begin{array}{l}\text { Obtener frecuencia cardiaca } \\
\text { en reposo inferior a } 80 \\
\text { latidos por minuto }\end{array}$ & Ila & B \\
\hline $\begin{array}{l}\text { Usar amiodarona intravenosa } \\
\text { para el control de la } \\
\text { respuesta ventricular en } \\
\text { pacientes críticos sin } \\
\text { preexcitación }\end{array}$ & Ila & B \\
\hline $\begin{array}{l}\text { La ablación del nodo } \\
\text { aurículo-ventricular y el } \\
\text { implante de marcapasos es } \\
\text { razonable si no se alcanza el } \\
\text { control de la respuesta } \\
\text { ventricular con tratamiento } \\
\text { médico }\end{array}$ & Ila & B \\
\hline $\begin{array}{l}\text { Si coexiste preexcitación no se } \\
\text { deben utilizar } \\
\text { calcioantagonistas, } \\
\text { amiodarona o digoxina }\end{array}$ & III & B \\
\hline
\end{tabular}

baja o ha sido fallida. Cuando se utiliza esta estrategia se mejora la calidad de vida, se reducen las hospitalizaciones y en muchos casos se corrige la disfunción ventricular con recuperación dramática de la función sistólica. No se hace necesario continuar con medicamentos para controlar la respuesta ventricular, pero sí proseguir con anticoagulación. Cuando se va a realizar ablación del nodo se debe considerar la fracción de eyección previa al procedimiento y en caso de estar deteriorada, así sea en forma leve, se recomienda que el dispositivo a implantar tenga estimulación biventricular pues tiene beneficios clínicos para el paciente, como se demostró en el estudio BLOCK HF (Biventricular Versus Right ventricular Pacing in Heart Failure Patients With Atrioventricular Block) ${ }^{11}$ (tabla 2).

\section{Conflicto de intereses}

Los autores declaran no tener ningún conflicto de intereses.

\section{Bibliografía}

1. Halsey C, Chugh A. Rate versus rhythm control for atrial fibrillation. Heart Fail Clin. 2016;12:193-203.

2. Falk RH. Is rate control or rhythm control preferable in patients with atrial fibrillation? Rate control is preferable to rhythm control in the majority of patients with atrial fibrillation. Circulation. 2005;111:3141-50, discussion 57.

3. Holmqvist F, Kim S, Steinberg BA, et al. Heart rate is associated with progression of atrial fibrillation, independent of rhythm. Heart. 2015;101:894-9.

4. Daoud EG, Weiss R, Bahu M, et al. Effect of an irregular ventricular rhythm on cardiac output. Am J Card. 1996;78:1433-6.

5. Shinbane JS, Wood MA, Jensen DN, et al. Tachycardia-induced cardiomyopathy: a review of animal models and clinical studies. J Am Coll Cardiol. 1997;29:709-15.

6. Verma A, Newman D, Geist M, et al. Effects of rhythm regularization and rate control in improving left ventricular function in atrial fibrillation patients undergoing atrioventricular nodal ablation. Can J Cardiol. 2001;17:437-45. 
7. Freeman JV, Reynolds K, Fang M, et al. Digoxin and risk of death in adults with atrial fibrillation: the ATRIA-CVRN study. Circ Arrhythm Electrophysiol. 2015;8:49-58.

8. Washam JB, Stevens SR, Lokhnygina Y, et al. Digoxin use in patients with atrial fibrillation and adverse cardiovascular outcomes: a retrospective analysis of the Rivaroxaban Once Daily Oral Direct Factor Xa Inhibition Compared with Vitamin K Antagonism for Prevention of Stroke and Embolism Trial in Atrial Fibrillation (ROCKET AF). Lancet. 2015;385:2363-70.
9. Van Gelder IC, Groenveld HF, Crijns HJ, et al. Lenient versus strict rate control in patients with atrial fibrillation. N Engl J Med. 2010;362:1363-73.

10. Wyse DG, Waldo AL, DiMarco JP, et al. A comparison of rate control and rhythm control in patients with atrial fibrillation. N Engl J Med. 2002;347:1825-33.

11. Curtis AB, Worley SJ, Chung ES, et al. Improvement in Clinical Outcomes With Biventricular Versus Right Ventricular Pacing: The BLOCK HF Study. J Am Coll Cardiol. 2016;67:2148-57. 